\title{
Why do patients default from follow-up at a genitourinary clinic?: a multivariate analysis
}

\author{
J D C Ross, A McIver, A Blakely, J Dalrymple, W Peacock, C Wallis
}

\begin{abstract}
Objective-Firstly to compare the proportion of patients defaulting from follow up at a genitourinary medicine clinic with those attending other hospital based clinics. Secondly to determine which factors are associated with non attendance at a city centre genitourinary medicine clinic. Methodology-The proportion of patients who defaulted at a genitourinary medicine clinic, a general medical clinic, a general surgical clinic and a dermatology clinic during March 1995 were compared. A multivariate logistic regression analysis was performed comparing attenders and non attenders at the genitourinary medicine clinic with respect to time of appointment, diagnosis, previous contacts with clinic staff, potential domestic commitments and patient demographics in a prospective case control study.

Results-The default rate at the genitourinary medicine clinic was $15 \%$ compared with $13 \%, 15 \%$ and $14 \%$ for medical, surgical and dermatology clinics respectively. Patients who defaulted from the genitourinary medicine clinic (167) were compared with 172 attenders and significant differences found for timing of appointments, area of residence, frequency of counselling by the health advisor and age of the patient. Other factors such as the diagnosis, whether a woman had children, sexual orientation, whether negative results had been given over the phone, source of referral, sex of patient, employment status and the weather were not found to be significantly associated with defaulting from an appointment.

Conclusions-The time of the appointment and being seen by a health advisor were the only variables identified over which the clinic has control and therefore could potentially reduce non attendance rates.
\end{abstract}

(Genitourin Med 1995;71:393-395)

Keywords: genitourinary medicine; sexually transmitted disease; default; attendance

\section{Introduction}

Failure of patients to attend for review appointments may result in the inefficient use of medical and nursing time and also has implications for the control of transmissible infections both for the individual and the community. The proportion of patients defaulting from London genitourinary medicine (GUM) clinics has been reported to be between $28 \%$ and $32 \%^{12}$ which is comparable to that seen in general practice where rates between $5 \%$ to $30 \%$ have been reported. ${ }^{3-5}$ Despite these relatively high default rates little is known about what determines whether patients attend or not. If such factors could be identified, and if these were reversible, there is the potential to reduce levels of non-attendance.

This study compares the default rate of patients who had previously attended a GUM clinic with that of other hospital clinics. To determine which factors are associated with patients' defaulting from follow up a comparison was made between defaulters and attenders at the GUM clinic using a multivariate model.

\section{Methods}

The default rate of review patients attending a city centre GUM clinic at Edinburgh Royal Infirmary was calculated as a percentage of total attendances and compared with that of general medical outpatients, general surgical outpatients and dermatology outpatients at the same hospital during March 1995.

A prospective case-control study design was used to compare the characteristics of patients who defaulted from follow up at a city centre GUM clinic with those who attended for review. Initial power studies suggested that to have an $80 \%$ chance of detecting a 2.5 fold difference between attenders and non attenders for a variable which occurred in $10 \%$ of attenders a sample size of 336 would be required. All patients who failed to attend for a review appointment over a 27 day period were included in the study. The control group were randomly selected from the GUM clinic list of those who had attended for a review appointment. Patients attending for the first time or with a new diagnosis were excluded from the analysis and only the first appointment was included for those who defaulted on more than one occasion within the study period.

A number of variables relating to time of appointment, diagnosis, previous contacts with clinic staff, potential domestic commitments and patient demographics were compared in the two groups using multiple logistic regression with backward conditional analysis on the SPSS for Windows package (SPSS Inc.) to determine the relevant factors.

\section{Results}

The default rates in review patients over a one month period in the GUM clinic, general 
Table 1 Proportion of patients defaulting from follow-up over 1 month

\begin{tabular}{llll}
\hline Clinic & No booked patients & No defaulters & \% age defaulters \\
\hline Genitourinary medicine & 1254 & 192 & $15 \%$ \\
Medical outpatients & 483 & 62 & $13 \%$ \\
Surgical outpatients & 1144 & 168 & $15 \%$ \\
Dermatology outpatients & 4157 & 590 & $14 \%$ \\
\hline
\end{tabular}

medical outpatients, general surgical outpatients and dermatology outpatients are shown in table 1 .

One hundred and sixty seven patients who had appointments for follow up at the GUM clinic defaulted over the study period. A control group of 172 patients were selected at random from the 914 patients who did attend for review over the same 27 day time period.

The variables included in the initial analysis and those found to be relevant in the final model following backward conditional analysis are shown in table 2. Time of appointment, area of residence, counselling by the health advisor and age were all found to be significant. Using the eight variables obtained in the final statistical model to predict which patients would attend or default would only correctly classify $65 \%$ of patients.

\section{Discussion}

The default rate observed in our GUM clinic population is not significantly different from that of other hospital departments although our patients almost certainly differ with respect to their age, referral source and diagnosis. The significant factors in predicting whether patients fail to attend for follow up include an afternoon appointment, residing

Table 2 Multivariate logistic regression analysis relating risk of defaulting with various factors

\begin{tabular}{|c|c|c|c|}
\hline Variables in initial model & No defaulting & No attending & $\begin{array}{l}\text { OR }(95 \% \text { CI) of } \\
\text { defaulting }\end{array}$ \\
\hline Afternoon appointment & 72 & 56 & $1.25(0.95-1.65)$ \\
\hline More than 4 previous attendances & 34 & 38 & $0.81(0.59-1 \cdot 1)$ \\
\hline Diagnosis of gonorrhoea & 4 & 3 & $1.31(0.51-3.33)$ \\
\hline Diagnosis of warts & 88 & 84 & $1.21(0.89-1.65)$ \\
\hline Diagnosis of chlamydia/NSU & 19 & 32 & $0.86(0.58-1.26)$ \\
\hline Negative HIV test performed & 13 & 17 & $0.89(0.57-1.37)$ \\
\hline D3 diagnosis made & 8 & 6 & $0.91(0.48-1.7)$ \\
\hline Last seen by doctor of same sex & 91 & 110 & $0.88(0.68-1.14)$ \\
\hline Woman with children & 18 & 30 & $0.9(0.61-1.35)$ \\
\hline Tuesday appointment & 43 & 32 & $0.96(0.65-1.42)$ \\
\hline Wednesday appointment & 15 & 26 & $0.8(0.51-1.26)$ \\
\hline Thursday appointment & 42 & 35 & $1.05(0.72-1.53)$ \\
\hline Friday appointment & 39 & 51 & $0.71(0.47-1.06)$ \\
\hline Homosexual & 19 & 17 & $0.92(0.57-1.5)$ \\
\hline Resident in Edinburgh & 116 & 128 & $0.55(0.35-0.87)$ \\
\hline Resident in Lothian (not Edinburgh) & 30 & 36 & $0.55(0.33-0.94)$ \\
\hline Negative results given on phone & 17 & 8 & $1.5(0.91-2.45)$ \\
\hline Seen by health advisor & 61 & 87 & $0.72(0.55-0.93)$ \\
\hline Self referred & 103 & 78 & $1.2(0.93-1.56)$ \\
\hline Male sex & 80 & 73 & $1.18(0.87-1 \cdot 6)$ \\
\hline Symptoms present at last visit & 129 & 136 & $1(0.72-1 \cdot 4)$ \\
\hline Over 5 weeks since last visit & 32 & 19 & $1.42(0.99-2.03)$ \\
\hline Aged under 25 years & 102 & 88 & $1.3(0.99-1.71)$ \\
\hline Unemployed & 76 & 65 & $1.22(0.95-1.56)$ \\
\hline Raining on appointment day & 15 & 13 & $1.19(0.73-1.95)$ \\
\hline \multicolumn{4}{|l|}{ Variables in final model } \\
\hline Afternoon appointment & 72 & 56 & $1.36(1.07-1.72)$ \\
\hline Resident in Edinburgh & 116 & 128 & $0.55(0.35-0.86)$ \\
\hline Resident in Lothian (not Edinburgh) & 30 & 36 & $0.55(0.33-0.9)$ \\
\hline Friday appointment & 39 & 51 & $0.8(0.61-1.04)$ \\
\hline Seen by health advisor & 61 & 87 & $0.73(0.57-0.93)$ \\
\hline Self referred & 103 & 78 & $1.27(1-1.61)$ \\
\hline Over 5 weeks since last visit & 32 & 19 & $1.38(1-1.91)$ \\
\hline Aged under 25 years & 102 & 88 & $1.37(1.09-1 \cdot 74)$ \\
\hline
\end{tabular}

further from the clinic, not having been previously seen by a health advisor and age under 25 years. Until the start of 1995 the clinic policy had been to bring return patients back in the afternoon and only those who could not attend at that time were seen in the morning. Although patients are now reviewed in both morning and afternoon it is possible that patients who have been attending for prolonged periods may also be those who tend, historically, to have afternoon appointments.

One limitation of the study was that patients were not asked why they had not attended. Contacting non-attenders directly may breach confidentiality and even if contacted the answers obtained would have to be interpreted with caution since patients may be unwilling to provide accurate explanations. In addition contact tracing in most clinics is successful in only around $60 \%$ of cases which suggests that such a strategy would not be very successful and would also lead to a large selection bias. Using the variables included in the final statistical model the chance of a patient attending or defaulting could be predicted accurately in only $65 \%$ of cases suggesting that other factors are also relevant. Non-attendance may also occur due to inefficient hospital administration and confusion about appointment times with some reports estimating that up to a third of missed attendances could be due to these factors. ${ }^{67}$

Although over 300 patients were included in the analysis the frequency of some factors was too small to be certain that a type II error (false negative) had not occurred. For example, a sample size of over 10000 would have been required to detect a $50 \%$ difference in attendance rates for patients with and without gonorrhoea owing to the low prevalence in our clinic population at present.

Previous work at a London hospital suggested that non-attendance was higher in those who had previously attended a GUM clinic and lower in those with a positive attitude to coming to a clinic, but in this study the numbers were small and neither factor reached statistical significance. ${ }^{1}$ Another London study, in 1985, found a higher default rate in heterosexual men and in those admitting to three or more partners in the preceding month but age, residence and presence of symptoms were not thought to be relevant. ${ }^{2}$ This study only looked at patients with gonorrhoea and the conclusions drawn from it are limited by potential selection bias (only half the eligible patients were analysed) and the univariate analysis method with the potential for confounding.

Additional health education from the clinic health advisor was associated with an improvement in subsequent attendance and others have also found that offering additional advice and emphasising the reasons for follow up can reduce the default rate. ${ }^{8}$ The provision of an information leaflet in isolation may not affect the overall default rate however. ${ }^{2}$

Studies performed in general practice suggest that young adults default more frequently ${ }^{3}$ and our data support this. Given the predomi- 
nantly young age of the GUM clinic population this may be a particularly relevant factor but unfortunately not one which is readily changed.

Our data suggest that a number of variables may influence the rate of non attendance at GUM clinics. Of these the timing of the appointment and review by a health advisor were the only factors which were amenable to change and patients who had appointments in the afternoon and had not previously seen the health advisor were twice as likely to default (OR $2 \cdot 3,95 \%$ CI $1 \cdot 28-4 \cdot 17$ ). Overall $44 \%$ of patients had been seen by a health advisor but, although there is scope to increase this proportion, this would have associated resource implications. Since this study was completed our clinic has started an evening appointment service and we intend repeating the study once this has become established.

1 Perfrement S, Overfield K. Which patients with sexually transmitted diseases default? $\mathrm{Br} \mathcal{F}$ Veneral Dis 1978; $54: 201-4$

2 Bewley $S$. Who defaults after treatment for gonorrhoea? Randomised controlled study of effect of an educational leaflet. Genitourin Med 1988;64:241-4.

3 Deyo RA, Inui TS. Dropouts and broken appointments. A eyo RA, Inui TS. Dropouts and broken appointments. A Care 1980;18:1146-57.

4 Oppenheim GL, Bergman JJ, English EC. Failed appointments: a review. $\mathcal{F}$ Fam Pract 1979;8:789-96.

5 Vikander T, Parnicky K, Demers R. New patient no-shows in an urban family practice centre: analysis and intervention. $\mathcal{F}$ Fam Pract 1986;22:263-8.

6 Mason C. Non-attendance at out-patient clinics: a case study. $\mathcal{f}$ Adv Nurs 1992;17:554-60.

7 Cosgrove $M$. Defaulters in general practice: reasons for default and patterns of attendance. $B r f$ Gen Prac 1990; default and

8 Goodrich GG. Gonococcal infection: the effect of educaGoodrich GG. Gonococcal infection: the effect of educa-
tional counselling on patient compliance. $B r f$ Venereal Dis $1981 ; 57: 137-40$. 